\title{
Response Tendency in a Questionnaire without Questions
}

\author{
J. van Heerden and Joh. Hoogstraten \\ University of Amsterdam
}

In a replication of an earlier study by Berg and Rapaport (1954), a questionnaire with items lacking content and containing merely answer possibilities was administered to 197 Dutch psychology freshmen. Students clearly did not react in accordance with the laws of statistical probability but showed, like Berg and Rapaport's subjects, a preference for positive options such as yes, true, and satisfied over negative options such as no, false, and dissatisfied. No sex differences or differences due to position of the answer possibilities were found. This result is in contrast with the 1954 findings, because Berg and Rapaport found that option position and subjects' sex did influence response bias.

A quarter of a century ago, Berg and Rapaport (1954) found that in a maximally unstructured and ambiguous situation, subjects showed a clear preference for some responses over others. Their research was based on the acknowledged psychological fact that the distribution of human reactions is biased, even in normal test situations. In order to show response bias, Berg and Rapaport constructed an "imaginary" questionnaire that lacked item content. It merely contained answer possibilities, such as yes, uncertain, no, or first, second, third. The results indicated that in a sit-

APPLIED PSYCHOLOGICAL MEASUREMENT

Vol. 3, No. 1 Winter 1979 pp. 117-121

(c) Copyright 1979 West Publishing Co. uation in which there were no actual questions, subjects still preferred certain responses to others. The bias was due mainly to preference for positive options over negative or neutral options. Berg and Rapaport believed that this positive bias could be explained by the American culture of the day, which valued positive attitudes in social situations. Other factors, such as position of the preferred answer or the sex of the subjects, probably influenced the bias; but these were clearly secondary in influence to content.

In this study, Berg and Rapaport's research was replicated at a later time and in quite a different situation. A few changes were also made in the procedure to avoid the possible contamination of examiners that Berg and Rapaport mentioned. Their possible influence was controlled by minimizing their role, as outlined below. The layout of the questionnaire was also varied. The answer possibilities to most items were not only presented in a horizontal but also in a vertical order. In addition, the questionnaire included a final question on the subject's feeling of certainty of the correct choice. The questions investigated were: Do response biases operate, as they did for Berg and Rapaport, even when questionnaire items have no content? Do these response biases operate similarly at a different time and in a different culture? 


\section{Method}

Eighty-eight female and 109 male first-year psychology students of the University of Amsterdam (almost the entire 1977-1978 population of freshmen) responded as part of their obligations to a questionnaire of 10 items. Students were asked to read the following instructions; the experimenter also read the statement aloud:

In this investigation we want to determine how well you can guess the answers to questions that are not known to you. On each of the following pages you will find a Roman numeral and a group of possible answers. Sometimes these alternatives are words; in other cases they are figures or characters. The question, however, is missing. You are to circle one of the alternatives, the one that you think is correct. You can be sure that in principle each of the options is a fitting response. Please work through the items in the order given. Try not to skip pages, and circle one alternative per item only. ${ }^{1}$

At this point, an item example with options black and red was given to emphasize the unusual character of the questionnaire. After that, students were requested to complete the 10 items. Each item was presented on a separate sheet and had four versions, differing in the relative position of the response alternatives. To illustrate, the options of Item IV were presented either horizontally (true-false; false-true) or, as in Berg and Rapaport's experiment, vertically

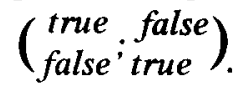

There were three exceptions, however; Item VIII was presented only in horizontal form with categories ranging from agree to disagree and with scale points ranging from $7 \ldots \ldots 1$ and $1 \ldots \ldots$ 7 , respectively. Item IX requested students to indicate their answers by a check mark on a 100

"The instructions and response alternatives were presented to subjects in Dutch. mm horizontal scale which had no appropriately labeled end points. This item was identical for all students. The final item, Item X, asked students to indicate on a 7 -point scale how certain they were of their answers to Item IX. The extremes of the scale (very certain-very uncertain) and the scale points $(1 \ldots \ldots 7,7 \ldots \ldots 1)$ were varied systematically. The allocation of the four versions to the total sample was random. Table 1 gives the items comprising the questionnaire ${ }^{2}$.

\section{Results}

The frequencies and percentages of response to the individual items obtained in the total sample $(N=197)$, and the results of $\chi^{2}$ tests to assess departures from chance expectation, are given in Table 1. The preferences demonstrated by Berg and Rapaport show a remarkable resemblance to those found in the current study. Items I, II, III, and V in particular resulted in almost identical distributions. Even the 1977 distribution of responses to items that did not indicate a clear-cut response bias (e.g., Items IV and VI) was highly similar to the distribution found 23 years earlier in the United States. In fact, there was only one exception to this pattern of congruency. In the present experiment, Item VIII showed a biased response favoring the indifferent, middle categories, whereas Berg and Rapaport's findings indicate a bias to the positive agree option. However, the present study made use of a version of this item that differed from the one used by Berg and Rapaport, since the students reacted to a 7 -point scale.

\footnotetext{
${ }^{2}$ Items VIII (agree $-1-\ldots \ldots \ldots$. . . disagree -7-) and X (very certain $-1-\ldots \ldots \ldots$ very uncertain -7.) were given as 7 -point alternatives. The distributions were as follows:

$\begin{array}{lrrrrrrr}\text { Options Item VIII } & 1 & 2 & 3 & 4 & 5 & 6 & 7 \\ \text { Frequencies of response: } & 21 & 27 & 35 & 46 & 36 & 17 & 15 \\ & & & & & & & \\ \text { Options Item X } & 1 & 2 & 3 & 4 & 5 & 6 & 7 \\ \text { Frequencies of response: } & 57 & 21 & 13 & 38 & 11 & 16 & 41\end{array}$
}


Table 1

Distribution of Choices in 1954 (Berg and Rapaport) and in 1977

\begin{tabular}{|c|c|c|c|c|c|c|c|}
\hline \multirow[b]{2}{*}{ Items } & \multirow[b]{2}{*}{ Alternatives } & \multicolumn{3}{|c|}{1954 (Regular Form) } & \multicolumn{3}{|c|}{1977} \\
\hline & & $\mathrm{N}$ & $\%$ & $x^{2}$ & $\mathrm{~N}$ & $\%$ & $x^{2}$ \\
\hline \multirow[t]{3}{*}{ I } & Yes & 82 & 48 & & 118 & 60 & \\
\hline & Uncertain & 43 & 25 & $16.5^{* * *}$ & 31 & 16 & $63.66 * * *$ \\
\hline & No & 46 & 27 & & 48 & 24 & \\
\hline \multirow[t]{4}{*}{ II } & 1 & 14 & 8 & & 21 & 11 & \\
\hline & 2 & 41 & 24 & $840 * * *$ & 53 & 27 & $6583^{* * *}$ \\
\hline & 3 & 92 & 54 & 84.0 & 95 & 48 & 03.03 \\
\hline & 4 & 24 & 14 & & 28 & 14 & \\
\hline \multirow[t]{4}{*}{ III } & very satisfied & 22 & 13 & & 47 & 24 & \\
\hline & Satisfied & 93 & 54 & $85.0 * * *$ & 89 & 45 & $44.56^{* * *}$ \\
\hline & Dissatisfied & 40 & 24 & & 32 & 16 & \\
\hline & $\begin{array}{l}\text { Very dissatis- } \\
\text { fied }\end{array}$ & 16 & 9 & & 29 & 15 & \\
\hline \multirow[t]{3}{*}{ IV } & First & 62 & 36 & & 81 & 41 & \\
\hline & Second & 59 & 35 & 1.3 & 70 & 36 & $9.21 * *$ \\
\hline & Third & 50 & 29 & & 46 & 23 & \\
\hline \multirow[t]{2}{*}{$\mathrm{V}$} & True & 118 & 69 & $239 * * *$ & 118 & 60 & $7.33^{* *}$ \\
\hline & False & 53 & 31 & $20-3$ & 79 & 40 & \\
\hline \multirow[t]{4}{*}{ VI } & A & 31 & 18 & & 54 & 28 & \\
\hline & B & 64 & 37 & $180^{* * *}$ & 56 & 28 & 6.15 \\
\hline & $\mathrm{C}$ & 47 & 28 & & 53 & 27 & \\
\hline & $\mathrm{D}$ & 29 & 17 & & 34 & 17 & \\
\hline \multirow[t]{3}{*}{ VII } & Always & & & & 52 & 26 & \\
\hline & Sometimes & & - & & 91 & 46 & $14.25^{* * *}$ \\
\hline & Never & & & & 54 & 28 & \\
\hline \multirow[t]{3}{*}{ VIII } & Agree & 72 & 42 & & 48 & 24 & \\
\hline & Indifferent & 52 & 30 & $6.1^{*}$ & 117 & 60 & $24.28^{* * *}$ \\
\hline & Disagree & 47 & 28 & & 32 & 16 & \\
\hline \multirow[t]{5}{*}{ IX } & $0-1.5 \mathrm{~cm}$ & & & & 29 & 15 & \\
\hline & $1.5-3.5 \mathrm{~cm}$ & & & & 48 & 24 & \\
\hline & $3.5-6.5 \mathrm{~cm}$ & & - & & 57 & 29 & 3.42 \\
\hline & $6.5-8.5 \mathrm{~cm}$ & & & & 31 & 16 & \\
\hline & $8.5-10 \mathrm{~cm}$ & & & & 31 & 16 & \\
\hline \multirow[t]{3}{*}{$\mathrm{X}$} & Very certain & & & & 78 & 40 & \\
\hline & Indifferent & & - & & 62 & 31 & $14.34^{* * *}$ \\
\hline & very uncertain & & & & 57 & 29 & \\
\hline
\end{tabular}

${ }_{* *}^{*} \mathrm{p}<.05$

** $\mathrm{p}<.01$ 
In order to assess the possibility that the response distributions were related to subjects' sex or the specific position of options, $\chi^{2}$ tests were computed for each item. For three items the number of categories had to be reduced by combining adjacent scale points, since the expected frequencies were sometimes less than 5 . Therefore, in the case of Item VIII, scale points 1 and 2 (agree); 3, 4, and 5 (indifferent); and 6 and 7 (disagree) were combined. As indicated in Table 1 , the same post-hoc reduction of alternatives was realized for Item $X$ and, although in a different way, for Item IX. None of the chi-square values were statistically significant at the .01 level. Only one chi-square value was significant at $p<.05$, the distributions of reactions to Item VII being a function of option position. In general, however, the data suggest that option content alone was responsible for the biases found.

\section{Discussion}

It is surprising that students are willing to make a choice among answer possibilities to questions whose meaning they obviously cannot grasp, since there were no questions. This cooperation is in accord with an earlier finding (van Heerden \& Hoogstraten, 1976) that psychology freshmen do not hesitate to answer questions that contain a logical impossibility or have options that do not fit. In the present study, all students cooperated fully. Only on Item X, in which the amount of certainty was requested, 10 out of the 197 students showed doubt by correcting their answers. It is also interesting that the choice patterns obtained in this sample were, generally speaking, indicative of positive aspects, such as yes versus no, satisfied versus dissatisfied, and true versus false. Other items, however, showed a clear-cut tendency to select the evasive option. For instance, the option indifferent was markedly preferred to agree and disagree. Thus, it appears that in three out of four cases that allow such a choice (Items I, III, V), students preferred a positive option that holds for all versions of order, by which the conclusion can be justified that option content was the main cause of the observed bias, although the answer pattern to Item VIII showed an unmistakable tendency to neutrality. This can not be said of Item VII because it is doubtful if in this case the answer possibilities could be considered as positive, neutral, and negative attitudes.

Berg and Rapaport deplored their lack of control of the sex of the examiners as a variable, thereby not anticipating the possible influence of their attractiveness. The present study used three male examiners and found no differences due to sex. Nor was any influcnce found due to the position of the answer alternatives, which was mentioned as a secondary cause of bias by Berg and Rapaport and by other investigators cited in their report. Furthermore, it appeared that option 3 was appreciably more popular in 1977 and in 1954 than categories 1,2 , and 4 and that for Item IV a bias occurred at option first. As for the final item, Item X, the present data indicated that although a large proportion of the students preferred the indifferent categories (3, 4 , and 5), an even larger number showed a preference for either very certain or very uncertain.

Berg and Rapaport suggested that the observed preference for the positive in their study of 1954 may be interpreted as a definite choice for the socially most valued attitude in the American culture of their day. They stressed the possibility that other cultures or subcultures may value neutrality or negativism. The congruence between the present findings in the Netherlands and theirs, however, does not support this suggestion.

Finally, it might be argued that in actual research, the applicability of a questionnaire lacking item content is minimal. Subjects, however, are often asked to react to a questionnaire on topics they do not understand or to which they are completely indifferent. Such situations show a certain similarity to the presentation of a questionnaire without questions. Even the reactions to regular questionnaires might be con- 
taminated by a preference for certain options over others irrespective of item content.

\section{References}

Berg, J. A., \& Rapaport, G. M. Response bias in an unstructured questionnaire. The Journal of Psychology, 1954, 38, 475-481.

van Heerden, J., \& Hoogstraten, Joh. Antwoordbereidheid van proefpersonen. De Psycholoog, 1976, 11, 99-104.

\section{Acknowledgments}

The authors thank Fred N. Kerlinger and P. Koele for their comments on an earlier draft of this article.

\section{Authors' Addresses}

J. van Heerden, Psychologisch Laboratorium, Universiteit van Amsterdam, Weesperplein 8, Amsterdam, The Netherlands; Joh. Hoogstraten, Psychologisch Laboratorium, Universiteit van Amsterdam, Weesperlein 8, Amsterdam, The Netherlands. 